\title{
Ionospheric plasma irregularities studied with Swarm satellites
}

\author{
Wojciech J. Miloch ${ }^{1, *}$, Yaqi Jin $^{1}$, Chao Xiong ${ }^{2}$, Guram Kervalishvili ${ }^{2}$, Andres Spicher ${ }^{1}$, Lasse \\ B.N. Clausen ${ }^{1}$, and Claudia Stolle ${ }^{2}$ \\ ${ }^{1}$ Department of Physics, University of Oslo, Box 1048 Blindern, 0316 Oslo, Norway \\ ${ }^{2}$ Helmholtz Centre Potsdam, GFZ German Research Centre for Geosciences, Telegrafenberg, 14473 \\ Potsdam, Germany
}

\begin{abstract}
To study and characterise the ionospheric plasma irregularities at all latitudes, one can employ in-situ measurements by satellites in polar orbits, such as the European Space Agency's Swarm mission. Based on the Swarm data, we have developed the Ionospheric Plasma IRregularities (IPIR) product for a global characterisation of ionospheric irregularities along the satellite track at all latitudes. This new Level-2 data product combines complementary datasets from the Swarm satellites: the electron density from the electric field instrument, the GPS data from the onboard GPS receiver, and the magnetic field data from the onboard magnetometers. This can be used as a new tool for global studies of ionospheric irregularities and turbulence.
\end{abstract}

\section{Introduction}

Ionospheric plasmas are often characterised by density irregularities. They are the result of various plasma instabilities, which reflect complex interactions between the solar wind, Earth's magnetosphere and ionosphere [2]. Plasma density irregularities and fluctuations can influence propagation of trans-ionospheric radio waves and are thus of importance for ground based operations that rely on precise positioning with Global Navigation Satellite Systems (GNSS) [4]. Indeed, ionospheric scintillations are one of the first observed space weather effects $[4,5]$. Understanding ionospheric plasma irregularities and fluctuations is thus of both scientific and practical interest.

In-situ measurements of plasma irregularities and fluctuations can be successfully carried out by the Swarm satellites with their comprehensive set of instruments. Combining data from different instruments allow for characterising plasma variations in the ionosphere along the Swarm trajectory at all latitudes. Through IPIR - Ionospheric Plasma IRregularities product we provide characteristics of plasma density structures in the ionosphere, in terms of their amplitudes, gradients and spatial scales, and can assign them to predominant plasma processes and regions in the ionosphere. This opens for extensive, global studies of plasma irregularities and fluctuations.

\footnotetext{
*e-mail: w.j.miloch@fys.uio.no
} 

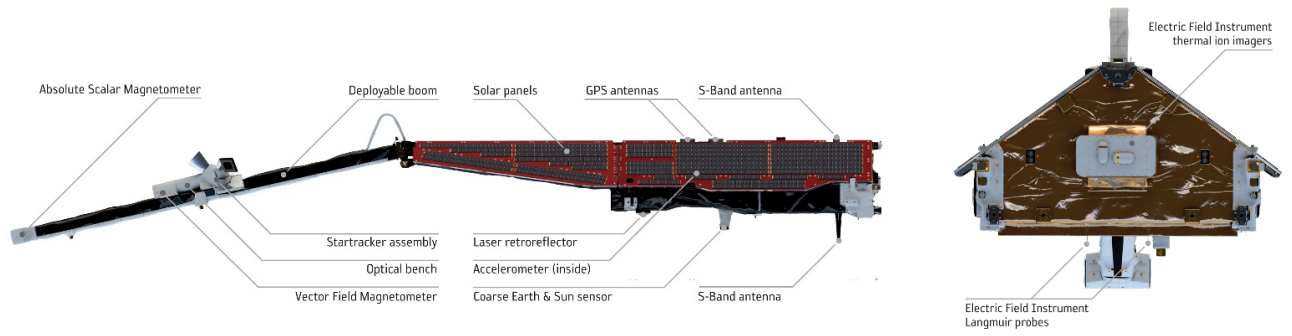

Figure 1. Side and front views of one of the Swarm satellites with the instrument placements. In IPIR we use mostly data from Langmuir probe, but also from magnetometers and GPS receiver. Source: European Space Agency, ESA/AOES Medialab.

\section{Swarm satellites}

Swarm is the European Space Agency's mission for studying Earth's magnetic field, upper atmosphere, and ionosphere (e.g., [1] [8]). Three identical satellites, denoted as A, B, and C, were launched in November 2013 into the polar orbits with an altitude of ca. $500 \mathrm{~km}$. Since April 2014, Swarm B has been moved to the orbit of $520 \mathrm{~km}$, while Swarm A and C fly side by side at a lower altitude of ca. $470 \mathrm{~km}$. The orbital planes of the Swarm B deviates slowly from the two other by 1.5 hours of local time per year.

The main payload of Swarm includes high precision magnetometers, GPS receivers, and the Electric Field Instrument (EFI) composed of Langmuir Probes and Thermal Ion Imager [3]. The instrument locations at Swarm are shown in Fig. 1. In IPIR, we use datasets from the Electric Field Instrument (EFI), GPS receivers and magnetometers.

Previously, the Swarm satellites have been successfully used for detecting the plasma bubbles (Ionospheric Bubble Index, IBI), which are irregularities in plasma occurring predominantly after sunset at low latitudes [6]. The data have also been used for the automatic detection of polar cap patches (Polar Cap Products, PCP), which are enhanced plasma density regions mostly poleward of the auroral oval, which can be associated with plasma instabilities [7]. Furthermore, the total electron content (TEC) derived from the on-board GPS receiver data, and the in situ measurements plasma density reveal similar characteristics [10]. In IPIR we combine those datasets and derive new quantities to characterise plasma density variations.

\section{Dataset description}

IPIR consists of 29 entries written in the CDF files. Most of them are related to the density measurements. We define two new parameters: The Rate of Change of Density $(R O D)$ and Rate of Change of Density Index (RODI). ROD is the temporal derivative of the plasma density calculated for the $2 \mathrm{~Hz}$ Swarm data:

$$
R O D(t)=\frac{N_{e}(t+\delta t)-N_{e}(t)}{\delta t}
$$

Where $\delta t=0.5 \mathrm{~s}$. This dataset is later on downsampled to $1 \mathrm{~Hz}$ data to have the compatibility with other datasets. The RODI10s and RODI20s are the standard deviation of ROD in a 
running window of $\Delta t=10 \mathrm{~s}$ or $\Delta t=20 \mathrm{~s}$, respectively:

$$
R O D I(t)=\sqrt{\frac{1}{N-1} \sum_{t_{i}=t-\Delta t / 2}^{t_{i}=t+\Delta t / 2}\left\|R O D\left(t_{i}\right)-\overline{R O D}\right\|^{2}},
$$

where

$$
\overline{R O D}=\sqrt{\frac{1}{N} \sum_{t_{i}=t-\Delta t / 2}^{t_{i}=t+\Delta t / 2} R O D\left(t_{i}\right)}
$$

While $R O D$ relates to the temporal variations in the density, which can be related to the smallest spatial scales measured by Swarm, we also calculate density gradients over the larger scales: 100, 50 and $20 \mathrm{~km}$, using a linear regression over a certain time interval. To provide information on the amplitudes of density fluctuations $\Delta N_{e}$, we derive the filtered electron density, where the median filtered value over 10, 20 and 40 seconds (corresponding to 75, 150 and $300 \mathrm{~km}$ scales) is subtracted from $N_{e}$. Finally we provide the background density calculated with the percentile filtering over scales of ca. $2000 \mathrm{~km} \mathrm{[7],} \mathrm{as} \mathrm{well} \mathrm{as} \mathrm{the} \mathrm{PCP} \mathrm{and}$ IBI flags for detecting plasma density structures at low and high geomagnetic latitudes.

The Total Electron Content (TEC), which is the line of sight integrated electron density between the Swarm and GPS satellites, is used to calculate the Rate of change of TEC (ROT): $R O T(t)=(T E C(t+\delta t)-T E C(t)) / \delta t$, with $\delta t=1 \mathrm{~s}$, and ROT Index (ROTI):

$$
\operatorname{ROTI}(t)=\sqrt{\frac{1}{N-1} \sum_{t_{i}=t-\Delta t / 2}^{t_{i}=t+\Delta t / 2}\left\|\operatorname{ROT}\left(t_{i}\right)-\overline{\operatorname{ROT}}\right\|^{2}},
$$

where $\Delta t=10 \mathrm{~s}$ or $\Delta t=20 \mathrm{~s}$, and $\overline{R O T}$ defined in a similar manner as in Eq. 3.

To assign the dataset to geomagnetic regions, we use the following scheme:

- Equatorial region corresponds to \pm 30 of Magnetic Latitude (MLAT),

- Mid-latitudes are regions between $\pm 30 M L A T$ and the corresponding equatorward Auroral Oval Boundary (AOB).

- Auroral oval region is determined in a dynamic way using the Field-Aligned Current (FAC) method based on the magnetic field measurements by Swarm [9].

- Polar Cap region is either poleward of the auroral oval, or when is not defined well at $>77^{\circ}$ MLAT.

Finally, we calculate the IPIR index, which is a parameter describing the severity of irregularities, where the high frequency fluctuations with large amplitudes are the most severe as they can have the largest space weather impacts such as affecting trans-ionospheric radio signals. The IPIR index is calculated as a product of RODI10s and a $A\left(N_{e}\right)$ which is the moving standard deviation of $\Delta N_{e_{10 s}}$ chosen in a running window of 10 seconds for consistency with $R O D I 10 s$. RODI is the variance of fluctuations in density and it indicates structuring of the plasma within the 10 seconds interval. On the other hand, $A\left(N_{e}\right)$ provides the absolute amplitude of fluctuations in plasma over a given time interval.

\section{Dataset examples}

In the following we present examples of a dataset for a selected day of 8 September 2017 to demonstrate how it can be used for studies of ionospheric plasma irregularities. In Fig. 2 we 


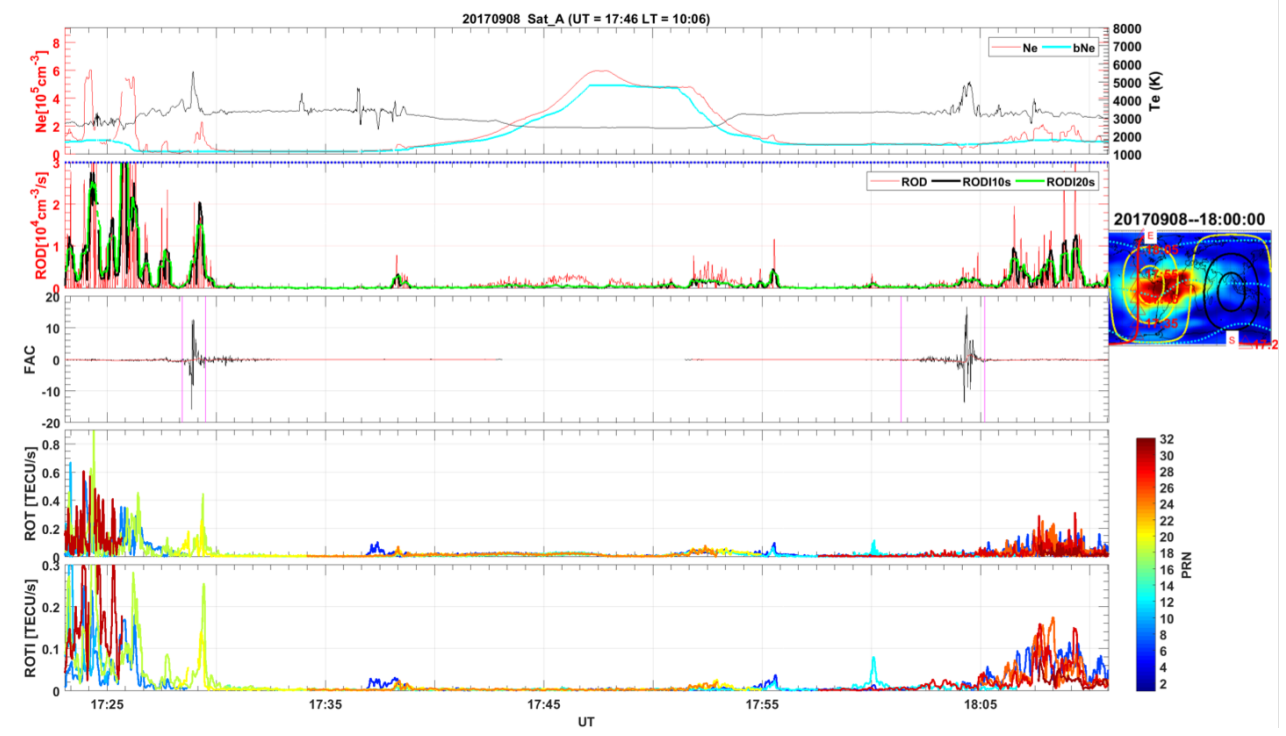

Figure 2. The local plasma density $N_{e}$, background density $b N e$, the electron temperature $T_{e}$, auroral oval markers, $R O D$ and RODI, as well as ROT and ROTI for September 8, 2017, during the pre-noon local time. Note that for clarity we plot the absolute values of density and TEC parameters.

present the results along the trajectory corresponding to the morning hours local time. The inset to the right of the panels shows the trajectory of Swarm over-plotted on the global TEC map for that particular day from the ground based receivers network. We note the enhanced plasma density around the equator, which is otherwise smooth, and no much variations in $R O D$ or $T E C$ are observed. However in the auroral oval and poleward of it, we observe high variations in both $R O D$ or $T E C$ indicating that plasma is structured at high latitudes. We see more plasma density fluctuations in the southern hemisphere for this particular pass (at the beginning of the time-series).

In Fig. 3, we show the trajectory corresponding to the post sunset local time for the same day. It is evident that the equatorial region ionospheric plasma is very irregular, which can be associated with post-sunset Equatorial Spread F and equatorial plasma bubbles. The auroral oval is larger than in Fig. 2, and the corresponding variations at high latitudes remain high at the southern hemisphere (end of time-series).

\section{Conclusions}

Based on Swarm data we have developed the Ionospheric Plasma IRregularities (IPIR) product for a global characterisation of ionospheric irregularities along the whole satellite track. This new Level-2 data product combines complementary datasets from the Swarm satellites: the electron density from the electric field instrument, the GPS data from the onboard GPS receiver, and the magnetic data, as well as IBI and PCP products. Finally it provides an index reflecting the strength of plasma irregularities, and assign irregularities to geomagnetic active regions. The IPIR dataset has been cross-validated, and selected parameters where validated against ground based measurements with the GNSS scintillation receivers at three different geomagnetic latitudes. IPIR provides 29 different parameters, and can be used as a 


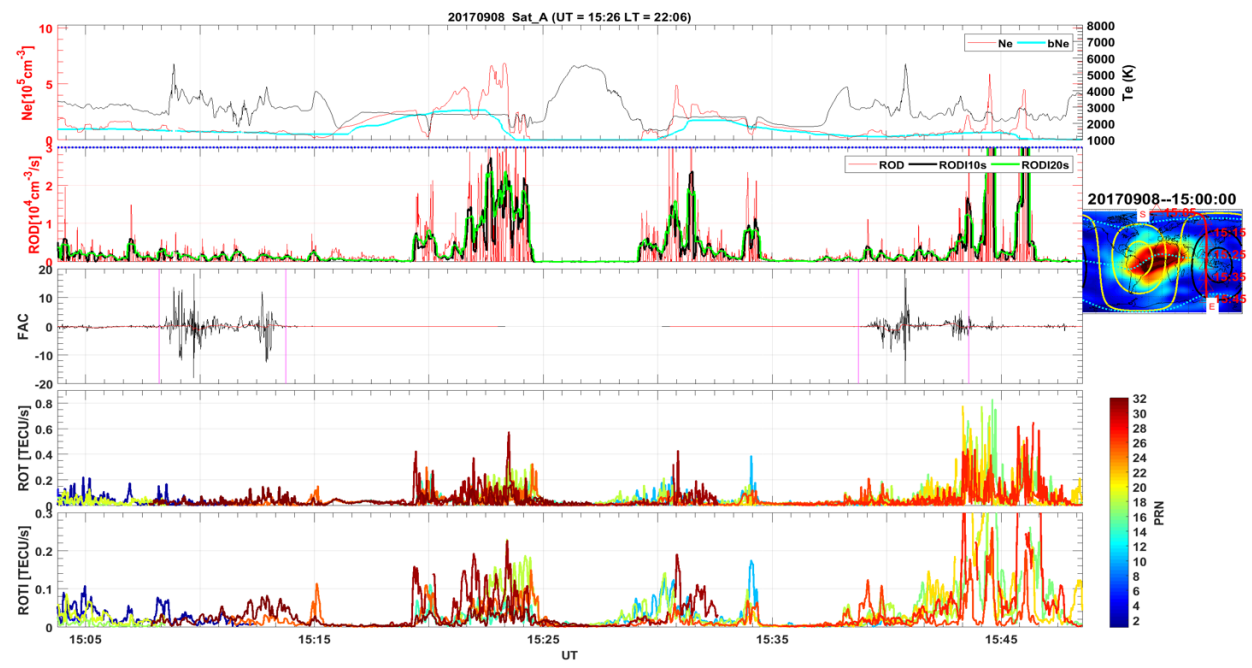

Figure 3. The same as in Fig.2 but for a pre-midnight local time.

new tool for global studies of ionospheric irregularities and turbulence. The data product can be downloaded from the ESA websites [11].

\section{Acknowledgments}

The research has been funded through ESA contract 4000109587/13/I-NB, as part of the Agency's Earth Observation Envelope Programme (EOEP). The GPS TEC data for GPS TEC map in Figs. 2 and 3. (insets) can be obtained through the Madrigal database at Haystack Observatory (http://madrigal.haystack.mit.edu/madrigal/). The Swarm data can be obtained through the official Swarm website: https://earth.esa.int/web/guest/swarm/data-access

\section{References}

[1] Friis-Christensen, E., H. Lühr, and G. Hulot, Earth, planets and space, 58(4), 351-35, (2006).

[2] Kelley, M. C. The Earth's Ionosphere Plasma Physics and Electrodynamics (Elsevier, Amsterdam, 2009).

[3] Knudsen, D. J., J. K. Burchill, S. C. Buchert, A. I. Eriksson, R. Gill, J.?E. Wahlund, L. Åhlen, M. Smith, and B. Moffat, J. Geophys. Res. Space Physics, 122, 2655?2673, (2017).

[4] Kintner, P. M., B. M. Ledvina, and E. R. de Paula, Space Weather, 5, S09003 (2007).

[5] Hey, J. S., S. J. Parsons, and J. W. Phillips, Nature 158(4007), 234-234 (1946).

[6] Park, J., M. Noja, C. Stolle, and H. Lühr, Earth Planets Space, 65(11), 1333-1344 (2013).

[7] Spicher, A., L. B. N.Clausen, W. J. Miloch, V. Lofstad, Y. Jin, and J. I. Moen, J. Geophys. Res., 122, 3837-3851 (2017).

[8] Stolle, C., R. Floberghagen, H. Luhr, S. Maus, D. J. Knudsen, P. Alken, E. Doornbos, B. Hamilton, A. W. P. Thomson, and P. N. Visser , Earth Planets Space, 65(11), 1375-1383, (2013). 
[9] Xiong, C., H. Luhr, H. Wang, and M. G. Johnsen, Ann Geophys-Germany, 32(6), 609622 (2014).

[10] Xiong, C., C. Stolle, and H. Lühr, Space Weather, 14(8), 563-577 (2016).

[11] https://earth.esa.int/web/guest/swarm/data-access, available from December 2018. 